\section{Reações adversas a medicamentos em pediatria: uma revisão sistemática de estudos prospectivos}

\section{Adverse drug reactions in pediatrics: a systematic review of prospective studies}

Djanilson Barbosa dos Santos 1

Helena Lutéscia Luna Coelho 1

1 Curso de Mestrado em Ciências Farmacêuticas Área de Concentração Farmácia Clínica. Universidade Federal do Ceará. Rua Capitão Francisco Pedro, 1210. Campus de Porangabussu. Fortaleza, CE, Brasil. CEP: 60.430-370

\begin{abstract}
The aim of this literature review is to explore and to compare information derived from prospective studies of adverse drug reactions (ADR) incidence in pediatrics. A systematic literature research was performed looking for studies involving outpatient and inpatient children as well hospitalizations due to ADR. The studies were identified in MEDLINE, IPA and LILACS databases from January 1966 to November 2003. Investigation focused on specific treatments or morbidities was not included in the review. Twenty prospective cohort studies were included in the analysis. In outpatient investigations ADR incidence varied from $0.75 \%$ to $11.1 \%$ between the studies; $0.6 \%$ to $4.3 \%$ of hospital admissions were related to $A D R$ with $A D R$ frequency during hospitalization varying from $4.4 \%$ to $18.1 \%$ of the children. The lack of definition standards and of methodological criteria hindered results comparison and could partly explain the different observations.

Key words Pharmaceutical preparations, Prospectives studies, Product surveillance, postmarketing Pharmacoepidemiology, Child
\end{abstract}

Resumo O objetivo desta revisão foi explorar e comparar as informações derivadas de estudos prospectivos sobre a incidência de reações adversas a medicamentos (RAM) em pediatria, publicados de janeiro de 1966 a novembro de 2003 em revistas indexadas nas bases MEDLINE, IPA e LILACS. Foram buscados trabalhos que abordassem a ocorrência de RAM em crianças atendidas em ambulatório, durante a hospitalização ou como causa de internação hospitalar não focalizados em tratamentos ou condições clínicas específicas. A análise incluiu vinte estudos de coorte prospectivos, entre os quais a incidência de RAM variou de 0,75\% a 11,1\% em ambulatório, de 0,6 a 4,3\% como causa de internação hospitalar e de 4,4 a 18,1\% entre crianças hospitalizadas. $O$ trabalho ressalta o potencial de contribuição dos estudos prospectivos para o uso seguro de medicamentos em pediatria e sugere que a padronização de definições e de critérios metodológicos poderia torná-los mais úteis para esse objetivo.

Palavras-chave Preparações farmacêuticas, Estudos prospectivos, Vigilância de produtos comercializados, Farmacoepidemiologia, Criança 


\section{Introdução}

A terapêutica farmacológica em crianças baseia-se, de maneira geral, na extrapolação das informações que levaram à aprovação do registro de medicamentos para uso em adultos. Esse fato compromete o resultado dos tratamentos e a sua segurança, como é reconhecido por diversos autores.1,2 Peculiaridades fisiológicas e farmacocinéticas nessa faixa etária se modificam ao longo do tempo, o que torna as crianças mais susceptíveis aos efeitos nocivos dos medicamentos e recomendariam uma atenção específica dos estudos de farmacovigilância. ${ }^{3-5}$ No entanto, em metaanálise publicada em 2001, Impicciatore et al.,6 revisando a literatura médica publicada entre 1966 e 2000 , identificaram apenas 21 estudos prospectivos sobre incidência de RAM em pediatria. A partir de 17 trabalhos selecionados para a análise estatística os autores calcularam em 9,53\% (IC95\%; 6,81-12,26) a incidência geral de RAM em crianças hospitalizadas e em 1,46\% (IC95\%; 0,7-3,03) naquelas atendidas em ambulatório; a frequiência média de RAM entre as causas de internação hospitalar foi calculada em $2,09 \%$ (IC95\%; 1,02-3,77). Ressalta-se no trabalho que $12 \%$ das RAM em crianças hospitalizadas e $39 \%$ das que causaram hospitalização foram fatais ou puseram em risco a vida dos pacientes.

Quanto aos fatores que explicariam essa elevada ocorrência de RAM em crianças, a citada revisão sugere o uso concomitante de vários medicamentos e o número médio de medicamentos administrados por criança. Em investigação sobre fatores associados a ocorrência de RAM em crianças hospitalizadas, Bonati et al.7 (1990) identificaram uma maior incidência em unidade de atenção intensiva neonatal (11 a 30\%), como também em crianças prematuras quando algum dos seguintes fatores está presente: idade gestacional inferior a 28 semanas, apnéia, uso de ventilação mecânica, nutrição parenteral total, insuficiência renal e/ou hepática.

Um aspecto adicional evidenciado em alguns trabalhos associa uma maior freqüência de RAM ao uso em crianças de medicamentos não licenciados para uso pediátrico (unlicensed) bem como ao emprego de produtos de maneira diversa das especificações autorizadas no registro (off label").8-11

A escassez de publicações sobre incidência de RAM em pediatria, bem como a heterogeneidade dos trabalhos publicados, particularmente no que se refere à metodologia e às variáveis registradas, limitam a extrapolação dos resultados para outras realidades, $o$ que justifica a presente revisão sistemática. Nela, procuramos ampliar as bases de dados consultadas na revisão mencionada, 6 com o intuito de buscar estudos realizados em outras realidades que não a européia ou dos EUA. O objetivo final do trabalho foi contribuir para a prática dos estudos de farmacovigilância em pediatria de modo a tornar os resultados mais úteis à promoção do uso seguro de medicamentos.

\section{Métodos}

Foi realizada uma revisão sistemática dos estudos publicados sobre a ocorrência de reações adversas a medicamentos em pediatria de janeiro de 1966 a novembro de 2003, período coberto pelo conjunto de bases de dados disponíveis.

\section{Critérios de inclusão e exclusão dos estudos}

Foram incluídos na revisão estudos observacionais descritivos ou analíticos de seguimento de reações adversas a medicamentos em pediatria, sem restrição de idioma de publicação, que apresentassem o cálculo da incidência acumulada de RAM, mesmo quando relacionada a cursos de tratamento. Foram excluídos os estudos focalizados em grupos farmacológicos e patológicos específicos, bem como as investigações retrospectivas ou que não apresentassem cálculo de incidência acumulada ou proporção de incidência.

\section{Estratégia de pesquisa e procedimento de revisão}

Foi realizada uma revisão sistemática para identificar estudos que atendessem aos critérios de inclusão estabelecidos. Para isso, foram pesquisados os bancos de dados Medline, de janeiro de 1966 a novembro de 2003, International Pharmaceutical Abstracts (IPA), de janeiro de 1966 a novembro de 2003 e Literatura Latinoamericana y del Caribe en Ciências de la Salud (LILACS), de janeiro de 1982 a novembro de 2003. Os descritores utilizados foram: "adverse drug reaction", "drug therapy/ adverse effects", "hospitalized children", "paediatric hospital", "hospitalized paediatric patients", "reação adversa a medicamento", "farmacoterapialefeitos adversos", "crianças hospitalizadas", "hospital pediátrico", "pacientes pediátricos hospitalizados". As referências dos estudos selecionados através da pesquisa nos bancos de dados foram examinadas em busca de artigos que atendessem aos critérios de seleção. Completou-se a revisão através de minuciosa análise da bibliografia contida nos artigos obtidos pelo método acima.

Os dados de cada estudo foram coletados independentemente por dois revisores através de formulários pré-delineados que incluíam variáveis correspon- 
dentes aos critérios de elegibilidade, variáveis referentes aos pacientes envolvidos nos estudos (idade, medicamentos utilizados), variáveis estatísticas (quiquadrado e teste de tendência) e dados sobre as reações adversas (imputabilidade, gravidade, órgãos e sistemas mais afetados, grupos farmacológicos mais envolvidos e fatores de risco).

\section{Resultados}

A pesquisa eletrônica inicial identificou no Medline do Pubmed 413, no Lilacs 6 e no IPA 126 artigos publicados sobre o tema "reações adversas a medicamentos em pediatria" no período de janeiro de 1966 a novembro de 2003. Vinte e dois estudos foram selecionados, dois foram excluídos, um porque focalizava apenas pacientes pediátricos de hematologia oncológica, 12 e outro porque não apresentava informações suficientes para se estimar a incidência de RAM. 13 Vinte trabalhos satisfizeram os critérios de inclusão, dos quais onze (Tabela 1) envolveram crianças hospitalizadas, cinco (Tabela 2) focalizavam RAM como causa de internação hospitalar e quatro (Tabela 3) tratavam de crianças atendidas em ambulatório. Todas as investigações selecionadas tiveram um delineamento observacional do tipo analítico prospectivo; quatro foram realizadas nos Estados Unidos, quatro na Espanha, três na Grã-Bretanha, duas na França, duas no Canadá, uma na Escócia, uma no Chile, uma em Israel, uma na Austrália e uma na Itália. A maior parte dos trabalhos abordou a incidência de RAM em crianças hospitalizadas (11/21) (Tabela 1). Em conjunto, os estudos cobriram uma população de 37.267 pacientes, variando de 260 a 7890 pacientes $(\bar{X}=1774,6$; IC95\%: 849,4-2699,8), sendo $20,7 \%$ hospitalizados. A duração total das investigações foi de 335 meses ( 28 anos), variando de 1 a 123 meses, sendo a média de 18,6 meses (IC95\%: 3,6-33,6). A idade dos pacientes variou de 8,5 a 99 meses, sendo a média de 43,9 meses (DP $=32,2$; mediana $=39,0$ meses; IC95\%: 20,8 - 66,9).

\section{RAM em crianças hospitalizadas (Tabela 1)}

A duração total dessas investigações foi de 128 meses ( 1 mês a 36 meses; $\bar{X}=11,6$; DP $=10,8)$, incluindo 7718 pacientes (260 a 1669 crianças; $\bar{X}=701,6$; $\mathrm{DP}=$ 414,7). A idade dos pacientes foi relatada em seis estudos, variando de 8,5 a 81,6 meses $(\bar{X}=43,2$ meses; $\mathrm{DP}=28,5$ meses; mediana $=45,6$ meses $)$. A incidência média de RAM foi de $10,1 \%$ (4,4\% a $18,1 \%)$. Sete estudos classificaram RAM quanto à causalidade e a proporção de reações na categoria "provável" variou de $32 \%$ a $100 \%$. A gravidade das reações foi registrada em seis artigos, sendo que a frequiência de reações graves foi de $4,4 \%$ a $27,9 \%$. Em dois estudos foram apresentados casos de RAM que resultaram em óbito, num total de três casos fatais. 14,15 Em um estudo realizado em Liverpool as reações adversas foram associadas a $6 \%$ dos medicamentos prescritos sem registro (unlicensed) ou para indicações não aprovadas (off-label) em comparação a 3,9\% das prescrições dos medicamentos com registro (RR=1,55, IC95\%: 1,19-2,03).9

\section{RAM como causa de hospitalização (Tabela 2)}

A duração total das investigações foi de 175 meses (7 a 132 meses; $\bar{X}=58,3$; DP $=65,4)$. O tamanho total da amostra dos estudos foi de 13.202 pacientes (512 a 6546 crianças; $\bar{X}=2640 ; \mathrm{DP}=2477)$. A dos pacientes variou de 9,0 a 99,0 meses ( $\bar{X}=43,2$ meses; $D P=$ $63,6$ meses; mediana $=54,0$ meses $)$, somente dois estudos relataram a média de idade dos pacientes.16,17 A incidência de RAM entre as causas de internação hospitalar variou de $0,6 \%$ a $4,3 \%$. Em todos os trabalhos foi avaliada a relação de causalidade entre as exposições e as RAM, mas não foram explicitados os critérios adotados; a proporção de casos classificados na categoria Provável variou de $30 \%$ a $96 \%$. A gravidade das RAM foi avaliada em três trabalhos, ${ }^{17-20}$ sendo que em dois deles foi relatada a ocorrência de óbitos. ${ }^{18,20} \mathrm{~A}$ freqüência das hospitalizações induzidas por reações adversas a medicamentos variou com a idade em dois dos cinco trabalhos analisados, observando-se no primeiro o aumento da incidência até os cinco anos de idade, tendendo à estabilidade após essa idade ( $\chi^{2}$ para tendência 11,8; p <0,05); na segunda investigação a freqüência de admissão por causa de RAM foi maior para crianças maiores de seis anos do que até essa idade $(\mathrm{p}<0.01) .18,20$ 
Resumo de estudos prospectivos de reações adversas a medicamentos (RAM) em crianças hospitalizadas.

\begin{tabular}{|c|c|c|c|c|}
\hline Autor, ano de & País & $\begin{array}{c}\text { Número } \\
\text { de crianças }\end{array}$ & RAM (\%) & Classificação de RAM e observações \\
\hline & Período & Idade & Incidência & (segundo o autor) \\
\hline $\begin{array}{l}\text { McKenzie et al., } \\
1973.15\end{array}$ & $\begin{array}{l}\text { E.U.A. } \\
(2 / 1971-9 / 1971)\end{array}$ & $\begin{array}{l}658 \text { crianças } \\
\text { Idade não relatada }\end{array}$ & $70 / 658=10,6$ & $\begin{array}{l}70 \text { pacientes }(10,6 \%) \text { apresentaram } 115 \text { reações adversas } \\
\text { envolvendo } 134 \text { medicamentos / } 1 \text { reação fatal }\end{array}$ \\
\hline Whyte, 1977.27 & $\begin{array}{l}\text { Escócia } \\
10 \text { meses }\end{array}$ & $\begin{array}{l}844 \text { crianças } \\
\text { Idade não relatada }\end{array}$ & $51 / 844=6,0$ & $\begin{array}{l}119 \text { reações adversas envolvendo } 15 \text { medicamentos. } \\
28 \text { definida, } 78 \text { provável, } 13 \text { possível } \\
24 \text { graves, } 56 \text { moderada, } 37 \text { leve, } 2 \text { vida-ameaçada }\end{array}$ \\
\hline $\begin{array}{l}\text { Mitchell et al., } \\
1979.25\end{array}$ & $\begin{array}{c}\text { E.U.A. } \\
1974-1977\end{array}$ & $\begin{array}{l}1669 \text { crianças } \\
\text { Média: } 81,6 \text { meses }\end{array}$ & $280 / 1669=16,8$ & $\begin{array}{l}280(16,8 \%) \text { crianças apresentaram reações } \\
\text { causalidade / severidade não relatada }\end{array}$ \\
\hline $\begin{array}{l}\text { Choonara et al., } \\
1984.32\end{array}$ & $\begin{array}{l}\text { Liverpool } \\
6 \text { meses }\end{array}$ & $\begin{array}{l}268 \text { crianças } \\
\text { Idade não relatada }\end{array}$ & $15 / 268=5,6$ & $\begin{array}{l}15 \text { crianças apresentaram } 17 \text { reações adversas. } \\
(100 \%) \text { definida / provável / severidade não relatada }\end{array}$ \\
\hline $\begin{array}{l}\text { Vasquez De La } \\
\text { Villa, } 1989.26\end{array}$ & $\begin{array}{l}\text { Espanha } \\
12 \text { meses }\end{array}$ & $\begin{array}{l}597 \text { crianças } \\
\text { Faixa: } 1-8 \text { anos } \\
\text { Média: } 46 \text { meses }\end{array}$ & $26 / 597=4,4$ & $\begin{array}{l}28 \text { reações adversas envolvendo } 17 \text { medicamentos. } \\
10 \text { definida, } 9 \text { provável e } 9 \text { possível } \\
3 \text { graves, } 14 \text { moderada e } 11 \text { leve }\end{array}$ \\
\hline Gil, 1995.28 & $\begin{array}{l}\text { Liverpool } \\
2 \text { meses }\end{array}$ & $\begin{array}{l}909 \\
\text { Faixa: } 4 d-16 \text { anos }\end{array}$ & $63 / 899=7,0$ & $\begin{array}{l}76 \text { reações adversas envolvendo } 35 \text { medicamentos. } \\
7(9 \%) \text { definida, } 33(43 \%) \text { provável e } 36(48 \%) \text { possível } \\
8(10 \%) \text { graves, } 19(25 \%) \text { moderada e } 49(65 \%) \text { leve }\end{array}$ \\
\hline $\begin{array}{l}\text { Gonzáles M.G., } \\
1998.30\end{array}$ & $\begin{array}{c}\text { Chile } \\
1997 \\
12 \text { meses }\end{array}$ & $\begin{array}{l}290 \text { crianças } \\
\text { Faixa: } 1 \text { mês-16 anos } \\
\text { Média: } 45,6 \text { meses }\end{array}$ & $30 / 219=13,7$ & $\begin{array}{l}46 \text { reações adversas envolvendo } 24 \text { medicamentos. } \\
(13,6 \%) \text { definida, }(54,2 \%) \text { provável e }(32,2 \%) \text { possível } \\
(27,9 \%) \text { graves, }(20,9 \%) \text { moderada, }(51,2 \%) \text { leve }\end{array}$ \\
\hline $\begin{array}{l}\text { Martinez-Mir, } \\
1999.14\end{array}$ & $\begin{array}{l}\text { Espanha } \\
7 \text { meses }\end{array}$ & $\begin{array}{l}512 \text { crianças } \\
\text { Faixa: } 1-24 \text { meses } \\
\text { Média : } 8,5 \text { meses }\end{array}$ & $59 / 409=14,4$ & $\begin{array}{l}59 \text { crianças apresentaram } 68 \text { reações adversas. } \\
(2,9 \%) \text { definida, }(75 \%) \text { provável, }(16,2 \%) \text { possível }(52,9 \%) \\
\text { leve, }(39,7 \%) \text { moderada, }(4,4 \%) \text { graves }\end{array}$ \\
\hline Turner, 1999.9 & $\begin{array}{l}\text { Liverpool } \\
1996 \text { (3 meses) }\end{array}$ & $\begin{array}{l}1046 \text { admissões } \\
\text { Faixa: } 1 \text { dia - } 18 \text { anos }\end{array}$ & $116 / 1046=11,0$ & $\begin{array}{l}157 \text { reações adversas envolvendo } 207 \text { medicamentos. } \\
89(57 \%) \text { definida ou provável/ } 17(11 \%) \text { graves }\end{array}$ \\
\hline $\begin{array}{l}\text { Jonville-Béra, } \\
2002.33\end{array}$ & $\begin{array}{c}\text { França } \\
0,3 \text { meses }\end{array}$ & $\begin{array}{l}260 \text { crianças } \\
\text { Média: } 76,1 \text { meses }\end{array}$ & $6 / 227=2,6$ & $\begin{array}{l}6 \text { reações adversas envolvendo } 3 \text { medicamentos. } \\
\text { causalidade / severidade não relatada }\end{array}$ \\
\hline $\begin{array}{l}\text { Buajordet, } \\
2002.23\end{array}$ & $\begin{array}{l}\text { Canadá } \\
5 \text { meses }\end{array}$ & $\begin{array}{l}665 \text { admissões } \\
\text { Faixa: } 0 \text { - } 16 \text { anos } \\
\text { Média: } 32,4 \text { meses }\end{array}$ & $105 / 579=18,1$ & $\begin{array}{l}105 \text { crianças apresentaram } 246 \text { reações adversas. } \\
\text { causalidade / severidade não relatada }\end{array}$ \\
\hline
\end{tabular}


Resumo de estudos prospectivos de reações adversas a medicamentos (RAM) que induzem admissão em hospital pediátrico.

\begin{tabular}{|c|c|c|c|c|}
\hline Autor, ano de & País & $\begin{array}{c}\text { Número } \\
\text { de crianças }\end{array}$ & RAM (\%) & Classificação de RAM e observações \\
\hline & Período & Idade & IA & (segundo o autor) \\
\hline $\begin{array}{l}\text { McKenzie et al., } \\
1976.18\end{array}$ & $\begin{array}{l}\text { E.U.A. } \\
3 \text { anos }\end{array}$ & $\begin{array}{l}3556 \text { admissões } \\
\text { Idade não relatada }\end{array}$ & $72 / 3556=2,0$ & $\begin{array}{l}72 \text { reações adversas envolvendo } 140 \text { medicamentos. } \\
40(55,6 \%) \text { moderada, } 28(38,9 \%) \text { grave, } 4(5,5 \%) \text { fatal }\end{array}$ \\
\hline $\begin{array}{l}\text { Yosselson } \\
\text { Superstine et al., } \\
1982.19\end{array}$ & Israel & $\begin{array}{l}906 \text { admissões } \\
\text { Faixa: } 0 \text { - } 16 \text { anos }\end{array}$ & $29 / 906=3,2$ & $\begin{array}{l}29 \text { crianças admitidas com RAM. } \\
19(66 \%) \text { definida ou provável / } 12(45 \%) \text { graves }\end{array}$ \\
\hline $\begin{array}{l}\text { Mitchell et al., } \\
1988.20\end{array}$ & $\begin{array}{l}\text { E.U.A. } \\
1974-1985\end{array}$ & $\begin{array}{l}6546 \text { admissões } \\
\text { Faixa: } 0-15 \text { anos }\end{array}$ & $131 / 6546=2,0$ & $\begin{array}{l}66 \text { pacientes apresentaram } 114 \text { reações }(87 \%) \text { envolvendo } \\
7 \text { medicamentos. } \\
67(51 \%) \text { definida ou provável; } 2(1,5 \%) \text { fatal }\end{array}$ \\
\hline $\begin{array}{l}\text { Martinez-Mir et al., } \\
\text { 1996.17 }\end{array}$ & $\begin{array}{l}\text { Espanha } \\
7 \text { meses }\end{array}$ & $\begin{array}{l}512 \text { admissões } \\
\text { Faixa: } 1-24 \text { meses }\end{array}$ & $21 / 490=4,3$ & $\begin{array}{l}42 \text { reações adversas envolvendo } 40 \text { medicamentos . } \\
4,8 \% \text { definida, } 71,4 \% \text { provável, } 9,5 \% \text { possível e } 14 \% \\
\text { condicional } \\
1 \text { caso }(4,8 \%) \text { leve, } 12(57,1 \%) \text { moderada, } 8(38,1 \%) \text { grave }\end{array}$ \\
\hline $\begin{array}{l}\text { Easton et al., } \\
1998.16\end{array}$ & Austrália & $\begin{array}{l}1682 \text { admissões } \\
\text { Faixa: } 4 \mathrm{~m}-18 \mathrm{a} \\
\text { Média: } 9 \text { anos }\end{array}$ & $10 / 1682=0,6$ & $\begin{array}{l}10 \text { crianças admitidas com RAM. } \\
3(30 \%) \text { definida ou provável severidade não relatada }\end{array}$ \\
\hline
\end{tabular}

Tabela 3

Resumo de estudos prospectivos de reações adversas a medicamentos (RAM) em crianças ambulatoriais.

\begin{tabular}{|c|c|c|c|c|}
\hline Autor, ano de & País & $\begin{array}{c}\text { Número } \\
\text { de crianças }\end{array}$ & RAM (\%) & Classificação de RAM e observações \\
\hline & Período & Idade & IA & (segundo o autor) \\
\hline $\begin{array}{l}\text { Kramer et al., } \\
1985.21\end{array}$ & $\begin{array}{l}\text { Canada } \\
(1981-19822) \\
12 \text { meses }\end{array}$ & $\begin{array}{l}3181 \text { crianças } \\
\text { Faixa: } 2 d-18,9 \text { anos } \\
\text { Mediana: } 28,8 \text { meses }\end{array}$ & $473 / 4244=11,1$ & $\begin{array}{l}473 \text { tratamentos apresentaram } 534 \text { reações adversas. } \\
\text { (24) definida, } 176 \text { provável, } 244 \text { possível e } 83 \text { improvável / } \\
\text { severidade não relatada }\end{array}$ \\
\hline $\begin{array}{l}\text { Sanz et al., } \\
1987.31\end{array}$ & Espanha & $\begin{array}{l}1327 \text { crianças } \\
\text { Faixa: } 0-14 \text { anos }\end{array}$ & $10 / 1327=0,75$ & $\begin{array}{l}10 \text { crianças apresentaram reações adversas. } \\
8(80 \%) \text { definida ou provável / severidade não relatada }\end{array}$ \\
\hline $\begin{array}{l}\text { Cirko-Begovic et } \\
\text { al., } 1989.29\end{array}$ & $\begin{array}{l}\text { lugoslávia } \\
1986 \\
3 \text { meses }\end{array}$ & $\begin{array}{l}2359 \text { crianças } \\
\text { Faixa: } 0-7 \text { anos }\end{array}$ & $63 / 2296=2,7$ & $\begin{array}{l}63 \text { pacientes apresentaram reações. } \\
3(4,8 \%) \text { definida, } 53(84,1 \%) \text { provável, } 7(11,1 \%) \text { possível } \\
\text { / severidade não relatada }\end{array}$ \\
\hline $\begin{array}{l}\text { Menniti-Ippolito } \\
\text { et al., } 2000.22\end{array}$ & $\begin{array}{l}\text { Itália } \\
(4 / 1996-3 / 1997) \\
14 \text { meses }\end{array}$ & $\begin{array}{l}7890 \text { crianças } \\
\text { Faixa: } 0-14 \text { anos }\end{array}$ & $119 / 7890=1,51$ & $\begin{array}{l}119 \text { reações adversas foram relatadas. } \\
\text { Faixa etária: } 1-4: 48 \text { reações adversas } \\
\text { Causalidade não relatada / } 100 \% \text { leve }\end{array}$ \\
\hline
\end{tabular}




\section{Órgãos e sistemas mais afetados e grupos} farmacológicos mais envolvidos em RAM

Os órgãos e sistemas mais envolvidos nas RAM diagnosticadas em crianças hospitalizadas foram o sistema nervoso central e o aparelho gastrointestinal.23-27 No estudo envolvendo crianças hospitalizadas, a pele e o sistema cardiovascular foram os mais afetados. 28 Somente um trabalho relativo às internações hospitalares relatou o sistema nervoso central e o trato gastrointestinal como os mais afetados. 17 Nas investigações desenvolvidas em ambulatório, o trato gastrointestinal e a pele foram os mais envolvi$\operatorname{dos} .22,29$

A classe terapêutica mais freqüentemente implicada em suspeita de RAM em crianças hospitalizadas foi "Antiinfecciosos de Uso Sistêmico."9,24,26 Com exceção do trabalho realizado em hospital chileno, os medicamentos mais envolvidos em RAM foram: salbutamol, asparaginase, metilprednisona e fenobarbital. ${ }^{30}$ Nas investigações sobre RAM como causa de internação hospitalar, os grupos farmacológicos mais envolvidos foram "Antiasmáticos e Antibióticos de Uso Sistêmico."17 Este segundo grupo foi também o mais prevalente nas RAM desenvolvidas em ambulatório, estando associados a distúrbios gastrointestinais e erupções cutâneas. Os broncodilatadores, aos quais foram atribuídas diferentes manifestações sobre o SNC, foram o segundo grupo de maior frequiência nas RAM ocorridas em ambulatório. 21

\section{Discussão}

Apesar da busca exaustiva em bases de dados sem restrição de idiomas de publicação, e do largo período coberto pela revisão (trinta e sete anos) foram encontrados apenas vinte e dois trabalhos sobre o tema, dos quais apenas um foi realizado em país da América Latina (Chile) evidenciando o incipiente desenvolvimento da farmacoepidemiologia na região. Com relação à revisão de Impiciatore et al., 6 o presente trabalho, apesar de ter ampliado as bases de consulta com a inclusão do Lilacs, IPA e Scielo, identificou somente três novos estudos que atendessem aos critérios de inclusão.20,22,31 Procurou-se então fazer uma análise detalhada das características e limitações dos trabalhos encontrados, no intuito de contribuir para o desenvolvimento desse tipo de investigação em países latino-americanos.

Os diversos artigos analisados apresentaram características dos pacientes e dos medicamentos associados a RAM de uma amostra geograficamente variada de estudos observacionais prospectivos. Os resultados da análise devem ser interpretados levando-se em conta a heterogeneidade dos estudos selecionados, nos quais o que se entende por reação adversa às vezes não é explicitado ou difere, bem como o método de avaliação da causalidade ou de identificação dos casos. Tais limitações, associadas à ausência de parâmetros estatísticos essenciais em vários estudos não permitiram, por exemplo, a realização de uma meta-análise estatística.

Na seleção dos artigos não houve restrição quanto à metodologia empregada para identificar RAM, porém até mesmo entre aqueles estudos que utilizavam métodos semelhantes, a proporção de incidência de RAM variou significativamente. Essa heterogeneidade poderia ser parcialmente explicada por diferenças na quantidade e variedade de medicamentos aos quais as crianças foram expostas em cada estudo. Outras variáveis que influenciam a incidência de RAM ou a sua análise, tais como diagnóstico, co-morbidades, posologia, peso, altura e etnia não foram informados regularmente na maioria dos trabalhos e por isso não constaram na análise. Outro fator que poderia justificar as diferenças nas incidências encontradas é a variabilidade nos métodos de detecção de RAM - notificação espontânea, busca ativa de casos, entrevistas diretas com pacientes permitindo uma contribuição diferenciada da subjetividade dos avaliadores nos diferentes estudos.

Os resultados apresentados na maioria dos trabalhos analisados sugerem que, em pacientes hospitalizados, a frequiência de RAM aumenta com o tempo de hospitalização e com o número de medicamentos a que o paciente foi exposto. Por outro lado, a contribuição da faixa etária diferenciada das crianças envolvidas para uma maior incidência de RAM pode ser observada em crianças menores de dois anos de idade. 14

Os resultados deste trabalho evidenciam a escassez de publicações sobre o tema e a importância dos estudos de reações adversas a medicamentos em crianças menores de dois anos de idade, principalmente em recém-nascidos ( 0 a 28 dias), particularmente no Brasil, onde a pesquisa em farmacovigilância ainda é incipiente.

A inclusão de pacientes intoxicados e em tratamento oncológico também poderia explicar a ampla variação das incidências observadas em alguns trabalhos.9,14,24 Por outro lado, é provável que a presença de pacientes com diferentes graus de morbidade, tais como aqueles internados em unidade de terapia intensiva (UTI) ou em estado grave, tenha contribuído para a variabilidade dos valores de incidência observados, tais como $\mathrm{I}=5,6 \%$ e $\mathrm{I}=$ $7 \% .27,30$ A incidência de RAM em crianças foi 
comparada com aquela observada em adultos abaixo de 65 anos, hospitalizados, tendo sido menor no primeiro grupo $(I=13,7 \%$ versus $24 \%)$. Os autores atribuem essa diferença ao fato de que os adultos são hospitalizados com múltiplas doenças e recebem mais medicamentos do que os pacientes pediátri$\cos .29 \mathrm{Um}$ outro aspecto explorado evidenciou que as reações adversas em crianças hospitalizadas são mais freqüentes nas prescrições de medicamentos sem registro (unlicensed) ou para indicações não aprovadas (off-label) em comparação com o uso de medicamentos registrados e conforme a indicação. A percentagem de medicamentos off-label e unlicensed foi associada significativamente com o risco de $\operatorname{RAM}(p<0,001) .9$

A nosologia e o uso de medicamentos dentro e fora do hospital são consideravelmente diferentes, sendo que em pediatria a maior parte da utilização de medicamentos ocorre em ambulatório, portanto em condições menos controladas de uso. Apesar disso, a incidência de RAM em crianças atendidas em ambulatório foi inferior àquela verificada em crianças hospitalizadas $(0,75 \%$ a $2,7 \%$ versus $2,6 \%$ a 18\%). (Tabela 1 e 3). A diferença poderia ser explicada pela farmacoterapia mais intensa e uso simultâneo de medicamentos entre as crianças hospitalizadas, como também pela maior probabilidade de detecção de RAM nesse contexto.

\section{Órgãos e sistemas mais afetados} e grupos farmacológicos mais envolvidos em RAM

A detecção de reações adversas a medicamentos e seu diagnóstico diferencial dependem do grau de atenção do profissional de saúde para o problema, por isso, as manifestações mais evidentes tais como as reações dermatológicas, e aquelas mais referidas pelos pacientes, como os distúrbios gastrointestinais, tendem a ser mais diagnosticadas. Por outro lado, o perfil das RAM e a predominância do tipo de reação ou órgão afetado também estão associados à prevalência de uso de distintos grupos terapêuticos, tal como ocorre, por exemplo, no caso dos antimicrobianos e das manifestações gastrintestinais no contexto hospitalar.

\section{Fatores de risco}

Os estudos incluídos na presente revisão sistemática sugerem uma associação significativa entre número de medicamentos administrados e o risco da ocorrência de RAM em crianças. $9,25,27,28$ Em dois dos trabalhos que envolveram crianças hospitalizadas, o resultado da análise multivariada apontou para uma associação significativa entre o número de medicamentos administrados e o risco de apresentar RAM $(\mathrm{RR}=1,27, \mathrm{IC} 95 \%: 1,21-1,34, p<0,001) ; 9$ correlação semelhante também foi verificada através de análise de regressão linear. 14 A maior permanência dos pacientes no hospital também foi considerada um fator de risco para RAM em crianças hospitalizadas, conforme resultados de análise bivariada em vários trabalhos.14,25,27 Tal análise estatística também deu resultados positivos significativos para a correlação entre idade e incidência de RAM em pediatria. 31,32 A proporção de admissão hospitalar por RAM variou com a idade, sendo que pacientes de um mês a cinco anos apresentaram maior freqüência de RAM do que as outras faixas etárias $\left(\chi^{2}=30,55 ; p=\right.$ 0,01). 19 Verificou-se uma maior incidência em bebês hospitalizados de um a dois anos de idade $(p<0,05)^{27}$ e em investigação realizada em ambulatório, foi observado que os pacientes de idade inferior a um ano desenvolveram mais RAM do que os pacientes de idade superior. ${ }^{20}$ Apontou-se uma diferença significativa, em análise bivariada da freqüência de RAM entre os sexos, sendo maior em meninas do que em meninos $(\mathrm{RR}=1,66$, IC95\%: 1,03 - 2,52). 14

\section{Vieses / Limitações do estudo}

O presente trabalho foi uma tentativa de integrar os resultados de estudos de incidência de reação adversa a medicamentos em crianças. Uma grande dificuldade encontrada na seleção dos artigos foi a heterogeneidade dos métodos utilizados nos diferentes estudos. Essa é uma dificuldade comum às revisões sistemáticas, pois à medida que se introduz novos critérios de inclusão, ganha-se em especificidade mas perde-se em sensibilidade, comprometendo o objetivo de mostrar o panorama das publicações sobre um assunto e correndo o risco de excluir artigos relevantes para a compreensão do problema em estudo. A pesquisa da literatura, neste trabalho, envolveu a utilização dos bancos de dados computadorizados Medline do Pubmed, IPA e Lilacs. Todas as referências listadas nos artigos identificados foram examinadas na tentativa de se localizar possíveis artigos suplementares. Os resumos correspondentes foram lidos com cuidado para evitar falsos positivos (estudos irrelevantes identificados) e falsos negativos (estudos relevantes não encontrados). É possível, no entanto, que algum artigo que pudesse ser incluído na revisão tenha sido ignorado, mesmo que se considerem satisfatórias a abrangência e adequação das bases consultadas e dos descritores utilizados. 


\section{Conclusões}

As reações adversas são parte importante dos riscos aos quais estão expostos os usuários de medicamentos, particularmente as crianças. Investigações desenvolvidas em diferentes contextos culturais e sanitários estimaram a incidência de RAM nessa faixa etária. Por outro lado as RAM têm sido responsabilizadas por internações hospitalares de crianças, colocando em risco a vida das mesmas ou levando-as a óbito. O número de medicamentos administrados, a idade da criança e o tempo de internação hospitalar são fatores de risco reconhecidos em vários trabalhos.
A incidência de reações adversas a medicamentos (RAM) tem sido estimada através de estudos farmacoepidemiológicos prospectivos realizados em vários cenários da área pediátrica. Em virtude da variabilidade de métodos e de resultados, é difícil extrapolar com segurança esses dados para outros contextos, o que não invalida a riqueza das informações obtidas. Conclui-se sobre a relevância dos estudos prospectivos na identificação e prevenção de riscos do uso de medicamentos em pediatria e sobre a necessidade de otimizá-los para a obtenção de resultados mais efetivos.

\section{Referências}

1. Rodriguez W, Roberts R, Murphy D. Adverse drug events in children: the US food and drug administration perspective. Cur Ther Res 2001; 62: 711-23.

2. Bonati M. Epidemiologic evaluation of drug use in children. J Clin Pharmacol 1994; 34: 300-5.

3. Cote CJ. Is the "therapeutic orphan" about to be adopted? Pediatrics 1996; 98: 118-23.

4. Shirkey H. Therapeutic orphans [Editorial]. Pediatrics 1999; 104: 583-4.

5. Cuzzolin L, Zaccaron A, Fanos V. Unlicensed and off-label uses of drugs in pediatrics: a review of the literature. Fund Clin Pharmacol 2003; 17: 125-231.

6. Impicciatore $\mathrm{P}$, Choonara I, Clarrkson A, Provasi D, Pandolfini C, Bonati M. Incidence of adverse adverse drug reactions in pediatric in/out - patients: a systematic review and meta-analisis of prospective studies. Bri J Clin Pharmacol 2001; 52: 77-83.

7. Bonati M, Marchetti F, Zullini MT, Pistotti V, Tognoni G. Adverse drug reactions in neonatal intensive care units. Adv Drug Rea Acute Pois 1990; 9: 103-18.

8. Turner S. Unlicensed and off label drug use in paediatric wards: prospective study. Br Med J 1998; 316: 343-5.

9. Turner S, Nunn AJ, Fielding K, Choonara I. Adverse drug reactions to unlicensed and off-label drugs on paediatric wards: a prospective study. Acta Paediatr 1999; 88: 965-8.

10. Conroy S, Mcintyre J, Choonara I. Unlicensed and off label drug use in neonates. Arch Dis Child Fetal Neonatal 1999; 80: 142-5.

11. Conroy S, Choonara I, Impicciatore P. Survey of unlicensed and off label drug use in paediatric wards in European countries. Br Med J 2000; 320: 79-82.

12. Collins GE, Clay MM, Falletta JM. A prospective study of the epidemiology of adverse drug reactions in pediatric hematology and oncology patients. Am J Hosp Pharm 1974; 31: 968-75.

13. Dharnidharca VR, Kandoth PN, Anand RK. Adverse drug reactions in pediatrics with a study of in hospital intensive surveillance. Indian Pediatr 1993; 30: 745-51.
14. Martinez-Mir I, Garcia-López M, Palop V, Ferrer JM, Rubio E, Morales-Olivas FJ. A prospective study of adverse drug reactions in hospitalized children. Br J Clin Pharmacol 1999; 47: 681-8.

15. Mckenzie MW, Stewart RB, Weiss CF, Cluff LE. A pharmacist based study of the epidemiology of adverse drug reactions in pediatric medicine patients. Am J Hosp Pharm 1973; 30: 898-903.

16. Easton KL, Parsons BJ, Starr M, Brien J. The incidence of drug-related problems as a cause of hospital admissions in children. Med J Aust 1998; 169: 356-9.

17. Martinez-Mir I, Garcia-López M, Palop V, Ferrer JM, Rubio E, Morales-Olivas FJ. A prospective study of adverse drug reactions as a cause of admission to a paediatric hospital. Br J Clin Pharmacol 1996; 42: 319-24.

18. Mckenzie MW, Marchall GL, Netzloff ML, Cluff LE. Adverse drug reactions leading to hospitalization in children. J Pediatr 1976; 89: 487-90.

19. Yosselson-Supertimes G, Weiss T. Drug related hospitalization in pediatric patients. J Clin Hos Pharm 1982; 713: 195-199.

20. Mitchell AA, Lacouture PG, Sheehan JE, Kauffman RE, Shapiro S. Adverse drug reactions in children leading to hospital admission. Pediatrics 1988; 82: 24-9.

21. Kramer MS, Hutchinson TA, Flegel KM. Adverse drug reactions in general pediatric outpatients. J Pediatr 1985; 106: 305-10.

22. Menniti-Ippolito F, Raschetti R, Da Cas R, Giaquinto C, Cantarutti L. Active monitoring of adverse drug reactions in children. Lancet 2000; 355: 1613-4.

23. Buajordet I, Wesenberg F, Brors O, Langslet A. Adverse drug events in children during hospitalization and after discharge in a Norwegian university hospital. Acta Paediatr 2002; 91: 88-94.

24. Lawson DH. Drug surveillance. Problems and challenges (The Boston Collaborative Drug Surveillance Program). Pediatr Clin North Am 1972; 19: 117-29. 
25. Mitchell AA, Goldman P, Shapiro S, Slone D. Drug utilization and reported adverse reactions in hospitalized children. Am J Epidemiol 1979; 110: 196-204.

26. Vazquez de la Villa A, Luna del Castilho JD, Galdo MG, Puche CE. Reacciones adversas causadas por medicamentos en pediatria. An Esp Pediatr 1989; 31: 49-53.

27. Whyte J, Greenan E. Drug usage and adverse drug reactions in paediatric patients. Acta Paediatr Scand 1977; 66: 767-75.

28. Gill AM, Leach HJ, Hughes J, Barker C, Nunn AJ, Choonara I. Adverse drug reactions in a paediatric intensive care unit. Acta Paediatr 1995; 84: 438-44.

29. Cirko-Begovic A, Vrhovac B, Bakran I. Intensive monitor- ing of adverse drug reactions in infants and preschoo children. Eur J Clin Pharmacol 1989; 36: 63-5.

30. Gonzalez MG, Caroca CM, Paris E. Adverse drug reactions (ADRs) in hospitalized pediatric patients. A prospective study. Int J Clin Pharmacol Ther 1998; 36: 530-3.

31. Sanz E, Boada J. Adverse drug reactions in pediatric outpatients. Int J Clin Pharm Res 1987; 7: 169-172.

32. Choonara IA, Harris F. Adverse reactions in medical inpatients. Arch Dis Child 1984; 59: 578-580.

33. Jonville-Béra AP, Giraudeau B, Blanc P, Beau-Salinas F, Autret-Leca E. Frequency of adverse drug reactions in children: a prospective study. Br J Clin Pharmacol 2002; 53: 205-207.

Recebido em 23 de agosto de 2004

Versão final apresentada em 10 de outubro de 2004

Aprovado em 3 de novembro de 2004 